\title{
Estudo Comparativo de Algoritmos de RWA para Redes Ópticas Translúcidas
}

Santana, J. L.

Escola Politécnica de Pernambuco Universidade de Pernambuco 50.720-001 - Recife, Brasil jls@ecomp.poli.br
Pereira, H. A.

Grupo de Pesquisa em Radiometria, Departamento de Engenharia Elétrica, Centro de Engenharia Elétrica e Informática Universidade Federal de Campina Grande 58.429-900 - Campina Grande, Brasil helder.pereira@dee.ufcg.edu.br
Chaves, D. A. R.

Escola Politécnica de Pernambuco Universidade de Pernambuco 50.720-001 - Recife, Brasil daniel_rchaves@yahoo.com.br

Resumo A utilização de redes de computadores para estudo, trabalho e negócios vem crescendo de forma exponencial nos últimos anos. Assim, serviços de rede devem ter qualidade, para que as pessoas possam executar suas atividades de forma segura e adequada. Redes ópticas devem ser escaláveis, tolerantes a falhas, capazes de suportar grandes volumes de dados e prover alta velocidade nas transmissões, além de ter alcance físico extenso, atendendo aos requisitos para serviços de comunicação de longa distância. Para isso, é preciso utilizar algoritmos de roteamento que possam encontrar melhores rotas para a transmissão desse trafégo.

\footnotetext{
Abstract Recently, the use of computer networks for study, work and business has grown exponentially. Thus, network services must have quality, so people can do their activities safely and properly. All-optical networks are scalable, fault-tolerant and capable of handling large data volumes and provide highspeed transmissions, besides having extensive physical reach, meeting the current requirements for networking services. And so we need routing algorithms can find better routes for the transmission of such traffic.
} 


\section{Introdução}

Tanto atualmente como em épocas remotas, a necessidade do homem sempre foi de se comunicar. Esta comunicação precisa ser extremamente veloz e que percorra distâncias cada vez maiores. Um dos principais avanços tecnológicos, que ajudou de forma poderosa para que a comunicação antes citada fosse alcançada, foi o surgimento da rede mundial de computadores (internet) [1], nas décadas de 1970 e 1980. A internet oferece aos usuários uma possibilidade de comunicação praticamente ilimitada, fazendo que surja um número crescente de novas aplicações para serem utilizadas nessa nova tecnologia. Essas aplicações são cada vez mais complexas, aumentando a quantidade de informação que precisa trafegar pela rede, precisando alcançar distâncias sempre maiores, necessitando de uma maior demanda por largura de banda.

As tecnologias implantadas nas redes de comunicação não possuem recursos para suprir a demanda prevista. A solução mais viável para atender a essa crescente necessidade por largura de banda são as redes ópticas [2], devido à sua extrema eficiência no transporte de dados em altas taxas de transmissão. Uma rede de comunicação é dita óptica quando o meio físico, usado para a transmissão das informações entre os nós da rede, é composto por cabos de fibra óptica.

As redes ópticas são divididas em opacas, transparentes (ou totalmente ópticas) e translúcidas [3,4]. Nas redes opacas, a camada óptica da rede é utilizada apenas para a transmissão. Todas as operações inteligentes, como a comutação ou a gerência são realizadas por circuitos eletrônicos. Nessas redes, em cada nó intermediário, que interliga o nó origem ao nó destino do caminho óptico (lightpath), o sinal sofre uma conversão óptica-elétrica-óptica $(\mathrm{O} / \mathrm{E} / \mathrm{O})$ [1]. Já nas redes totalmente ópticas o sinal óptico trafega desde o nó origem até o nó destino no domínio óptico, sem sofrer conversão O/E/O e tampouco regeneração [1]. As redes translúcidas são arquiteturas de rede que podem utilizar ilhas de transparências, que são interligadas por regeneradores eletrônicos, ou alguns nós com capacidade de regeneração [3].

As técnicas de transmissão tradicionalmente usadas em fibras ópticas são técnicas de multiplexação por divisão no tempo (TDM - Time Division Multiplexing), em que a transmissão é feita num determinado comprimento de onda, e por divisão do comprimento de onda (WDM - Wavelength Division Multiplexing) que está ganhando uma rápida aceitação, pois permite que vários canais independentes compartilhem a mesma fibra óptica, proporcionando um melhor aproveitamento da banda de transmissão disponível. Porém, devido aos múltiplos canais de comu- nicação poderem ser transmitidos em uma única fibra, surgem dois problemas: (i) selecionar os enlaces que devem ser utilizados para transmitir dados entre um par de nós (origem e destino); (ii) selecionar comprimentos de onda disponíveis nos enlaces. A combinação desses dois problemas é chamada de roteamento e alocação de comprimento de onda (RWA - Routing and Wavelength Assignment). Esse artigo está dividido da seguinte forma: na Seção 2, descreve-se os algoritmos de roteamento utilizados nesse trabalho, os cenários de simulação são descritos na Seção 3, na Seção 4 são apresentados os resultados e por fim na seção 6 são feitas as conclusões do estudo.

\section{Algoritmos de Roteamento}

A tarefa do roteamento, em uma rede, consiste em encontrar um conjunto de enlaces que interligue dois nós com necessidade de se estabelecer uma conexão. Uma rede óptica é representada por um grafo em cujas arestas é associado um determinado custo. Existem vários custos possíveis que podem ser utilizados, dentre eles: disponibilidade de comprimentos de onda, distância, número de saltos (hops), por exemplo [4]. O problema de selecionar uma rota é normalmente resolvido da seguinte maneira: 1) representa-se a topologia da rede por um grafo, 2) calcula-se o custo de cada enlace (braço do grafo) utilizando-se alguma métrica (custo) preestabelecida e, 3) utiliza-se um algoritmo que encontra a rota de menor custo entre os nós que desejam realizar uma determinada conexão. Neste artigo os algoritmos utilizados para comparação de desempenho foram os de menor caminho (SP - Shortest Path) [5], caminho menos congestionado (LRW- Least Resistance Weight) [5], menor número de saltos $(\mathrm{MH}-\mathrm{Mi}$ nimum Hop)[5], menor relação sinal ruído (OSNR - Optical Signal-to-Noise Ratio)[5] e o algoritmo por série de potência (PSR - Particle Series Routing) [5].

\section{Redes Ópticas Translúcidas}

Nas redes translúcidas a determinação da rota, que o sinal óptico deve seguir ao longo da rede, deve ser feita levando-se em conta como a qualidade do sinal é impactada no nó destino, além de considerar a presença dos nós com capacidade de regeneração. Por isso, a decisão de roteamento deve ser feita considerando aspectos como: disponibilidade de comprimentos de onda nos enlaces, aspectos da camada física que afetam a qualidade dos sinais ópticos propagantes e a disponibilidade de recursos de regeneração nos nós intermediários da rota. Como o processo de roteamento deve levar em conta também o estado atual que a rede se encontra, o processo de verificação e computação de rotas assume uma característica global devido à dinâmica de operação sistêmica da rede [5,6,7]. 
Em situações práticas, devido ao acúmulo de ruído, ao longo da propagação do sinal óptico na rede, o sinal pode precisar passar pelo processo de regeneração eletrônica, tornando assim inviável sua propagação fim-a-fim no domínio óptico pela rede $[5,7,8,9]$. Portanto, um algoritmo de roteamento para redes translúcidas também deve se preocupar como melhor utilizar o recurso de regeneração disponível na rede, Com essa finalidade, os algoritmos de roteamento são comparados neste trabalho, refletindo seu desempenho no cenário das redes ópticas translúcidas, visto que foram idealizados para aplicação em redes transparentes.

\section{Cenários de Simulação}

Todas as simulações foram feitas com a rede Pacific Bell Modificada, ilustrada na figura1, de acordo com os seguintes cenários:

Primeiro cenário: Rede transparente com uma variação no numero de comprimento de ondas (12, 24 e 36), carga da rede variando de 40 a 140 Erlangs com passo de 10 de 10 em 10 unidades e um número de chamadas igual $10^{7}$.

Segundo cenário: Rede translúcida com número de nós translúcidos sendo $25 \%$ do total de nós da rede, com 12 regeneradores por nó translúcido e com o número de comprimentos de onda variando entre 12, 24 e 36, a carga da rede variando de 40 a 140 Erlangs, com passo de 10 e um número de chamadas igual a $10^{7}$.

Terceiro cenário: Rede translúcida com o número de nós translúcidos sendo $50 \%$ do total de nós da rede, com 12 regeneradores por nó translúcido e com o número de comprimentos de onda variando entre 12,24 e 36, a carga da rede variando de 40 a 140 Erlangs, com passo de 10, e um número de chamadas igual a $10^{7}$.

Quarto cenário: Rede translúcida com o número de nós translúcidos sendo $75 \%$ do total de nós da rede, com 12 regeneradores por nó translúcido, com o número de comprimentos de onda variando entre 12,24 e 36, a carga da rede variando de 40 a 140 Erlangs, com passo de 10 e um número de chamadas de $10^{7}$.

Quinto cenário: Rede opaca com o número de regeneradores sendo de 1000 por nó, com o número de comprimentos de onda variando entre 12, 24 e 36, a carga da rede variando de 40 a 140 Erlangs, com passo de 10 e um número de chamadas de $10^{7}$.

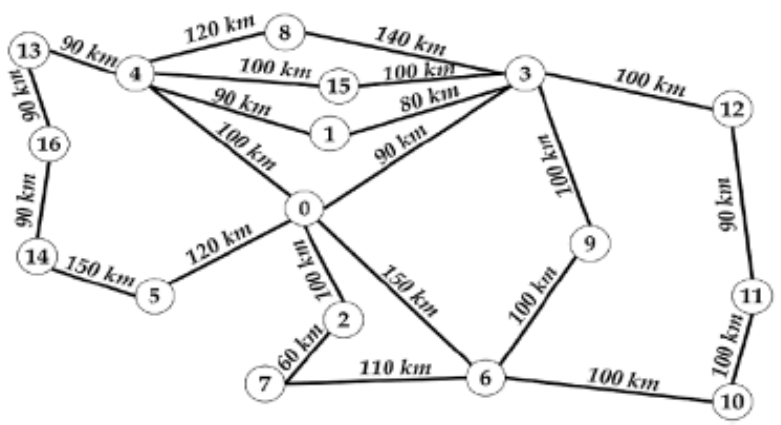

Figura 1 Topologia Pacific Bell Modificada.

\section{Resultados}

Para a rede transparente os algoritmos mostram resultados muitos próximos, porém o PSR e o OSNR obtiveram um melhor destaque, conforme ilustrado nas figuras 1(a), 1(b) e 1(c).

As figuras 2(a), 2(b) e 2(c), mostram os resultados para a rede com $25 \%$ de seu nós translucidos, obsernado-se assim que o algoritmo PSR obteve um melhor desempenho em relação aos outros algoritmos.

As figuras 3(a), 3(b) e 3(c), mostram os resultados para a rede com $50 \%$ de seu nós translucidos, obsernado-se assim que o algoritmo PSR obteve um melhor desempenho em relação aos outros algoritmos, porém para a figura 3(c), o algoritmo SP obteve um bom desempenho quando comparado com os outros, exceto PSR.

As figuras 4(a), 4(b) e 4(c), mostram os resultados para a rede com $75 \%$ de seu nós translucidos, obsernado-se assim que o algoritmo PSR obteve um melhor desempenho em relação aos outros algoritmos, porém para a figura 4(b) e 4(c), o algoritmo SP obteve um ótimo desempenho quando comparado com os outros, exceto PSR.

E para as figuras 5(a), 5(b) e 5(c) são mostrados os resultados para a rede opaca, obsernado-se que para este cenário o algoritmo PSR obteve um melhor desempenho em relação aos outros algoritmos que estão sendo analisados. 


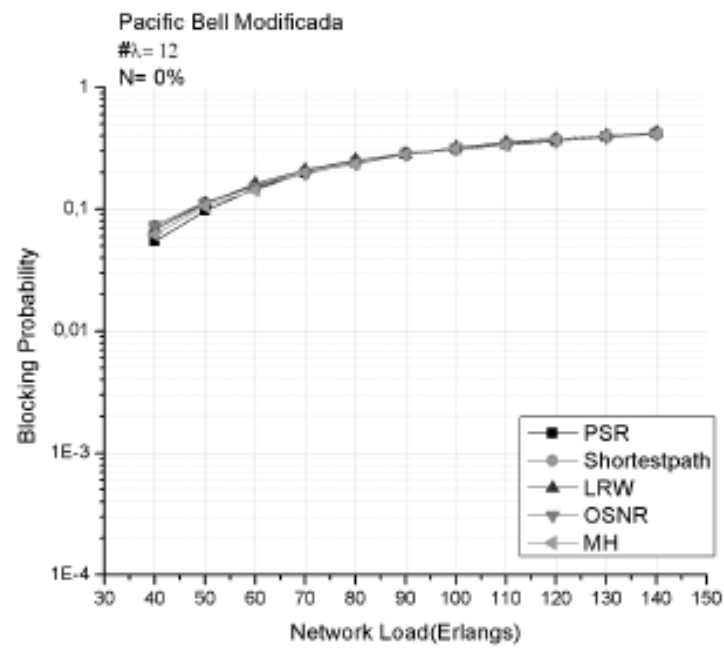

(a)

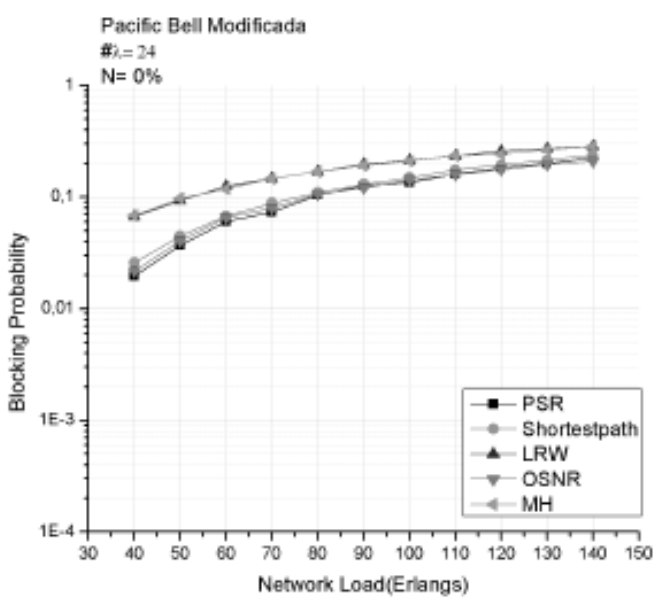

(b)

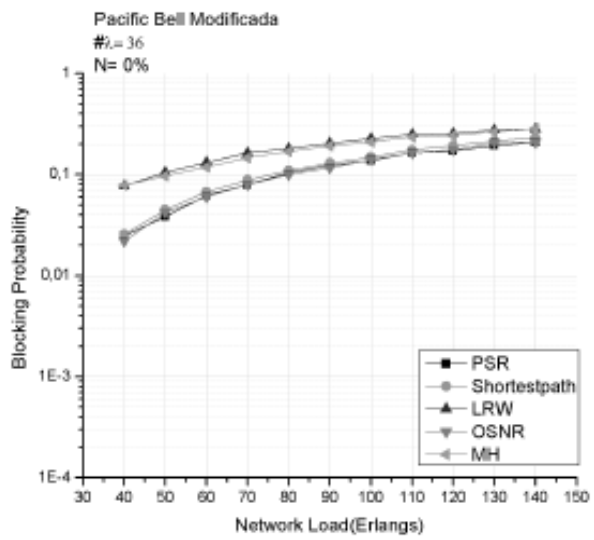

(c)
Figura 2: Topologia Pacific Bell Modificada considerando o cenário da rede transparente e: (a) 12 comprimentos de onda, (b) 24 comprimentos de onda e (c) 36 comprimentos de onda.

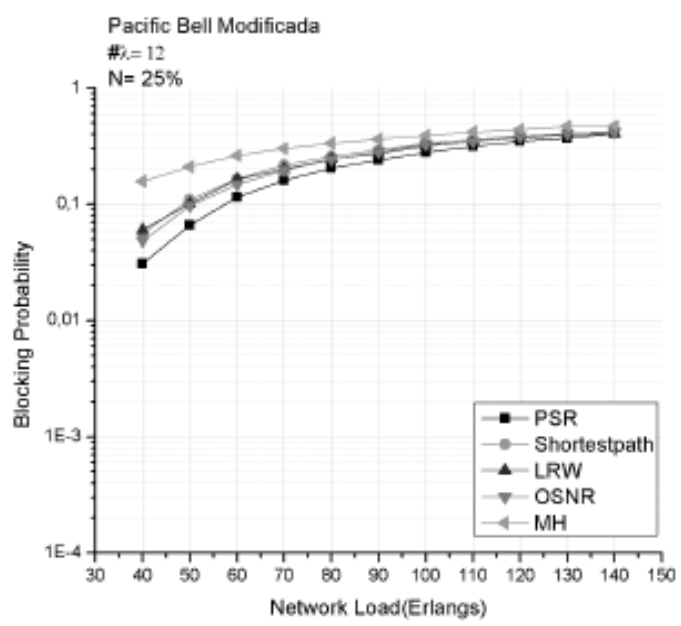

(a)

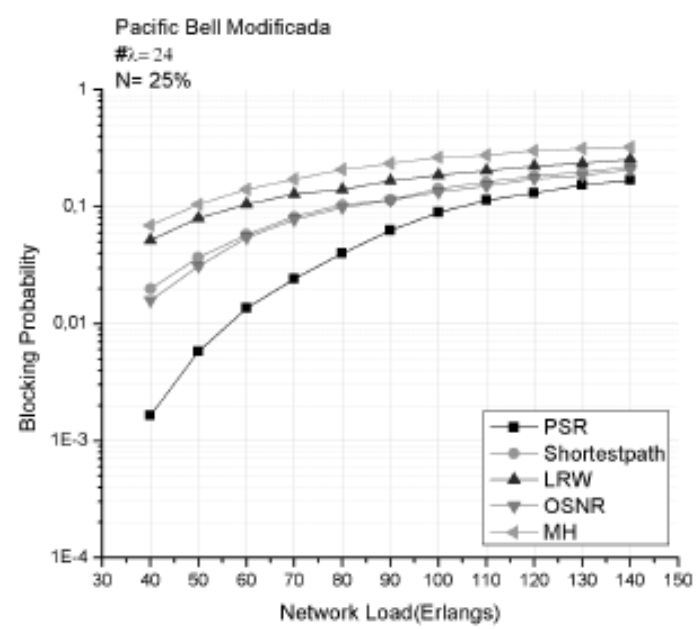

(b) 


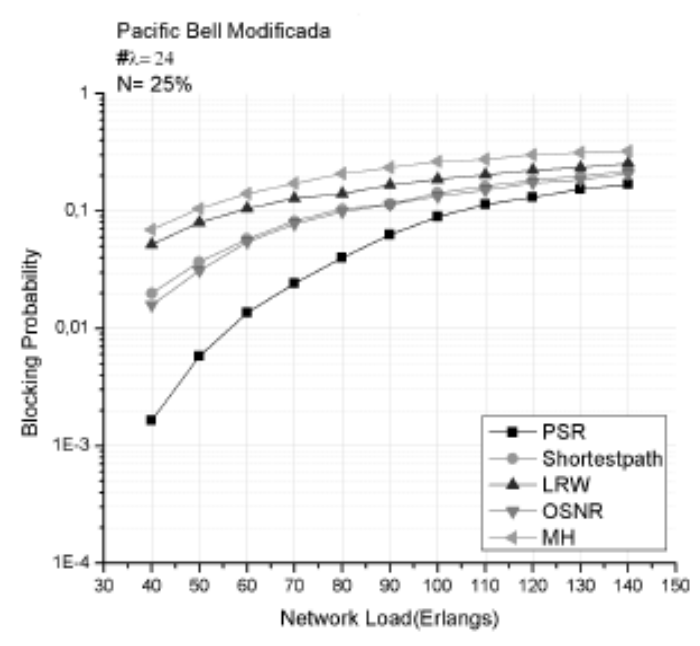

(c)

Figura 2: Topologia Pacific Bell Modificada considerando o cenário da rede translúcida com $25 \%$ dos nós translúcidos: (a) 12 comprimentos de onda, (b) 24 comprimentos de onda e (c) 36 comprimentos de onda.

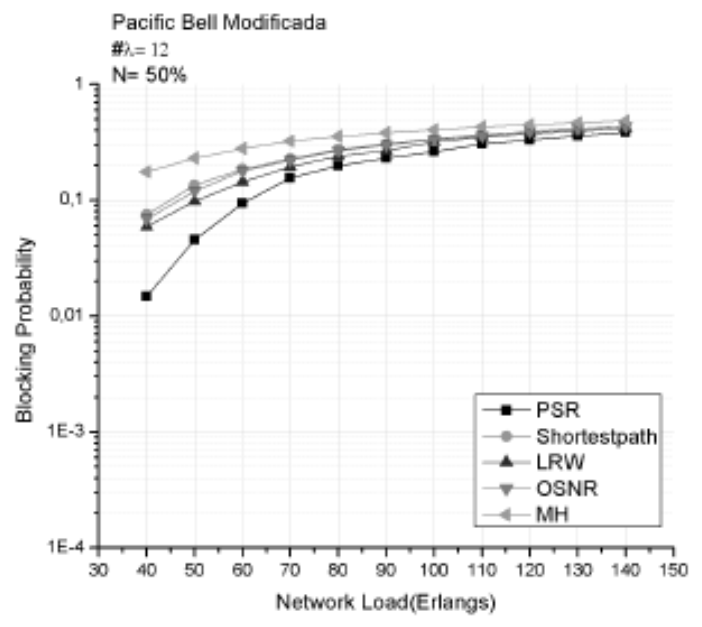

(a)

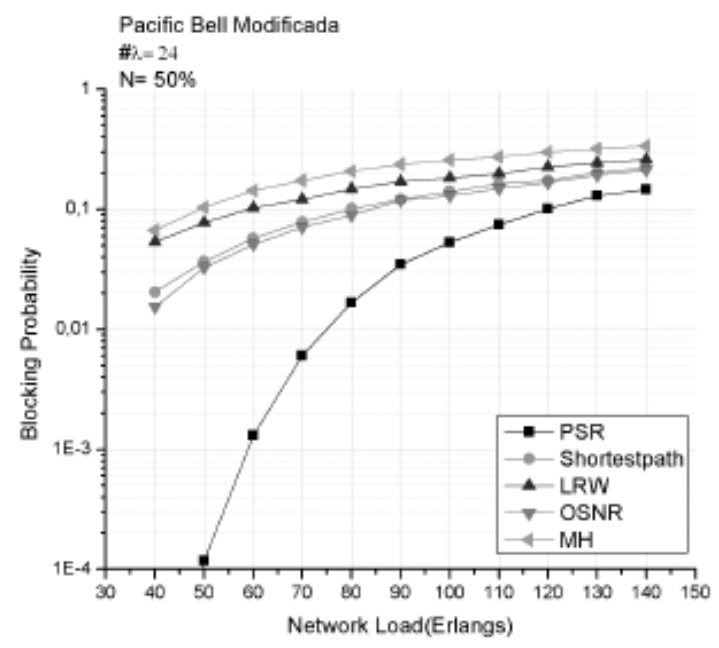

(b)

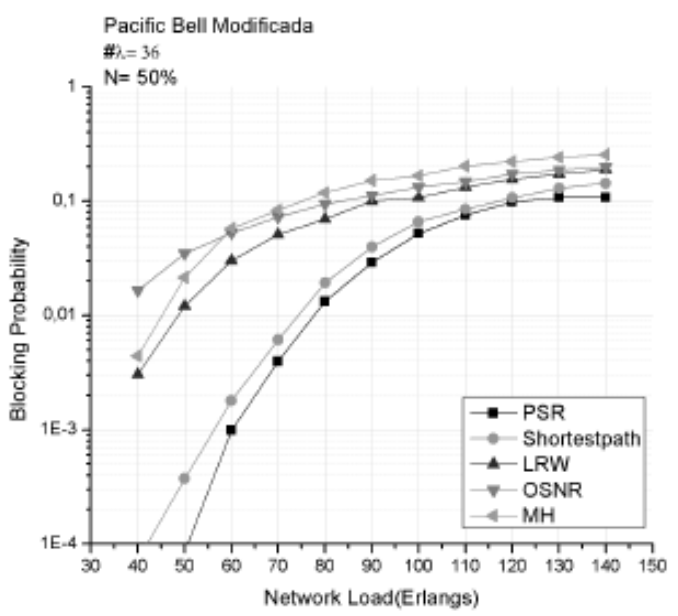

(c)

Figura 3: Topologia Pacific Bell Modificada considerando o cenário da rede translúcida com $50 \%$ dos nós translúcidos: (a) 12 comprimentos de onda, (b) 24 comprimentos de onda e (c) 36 comprimentos de onda. 


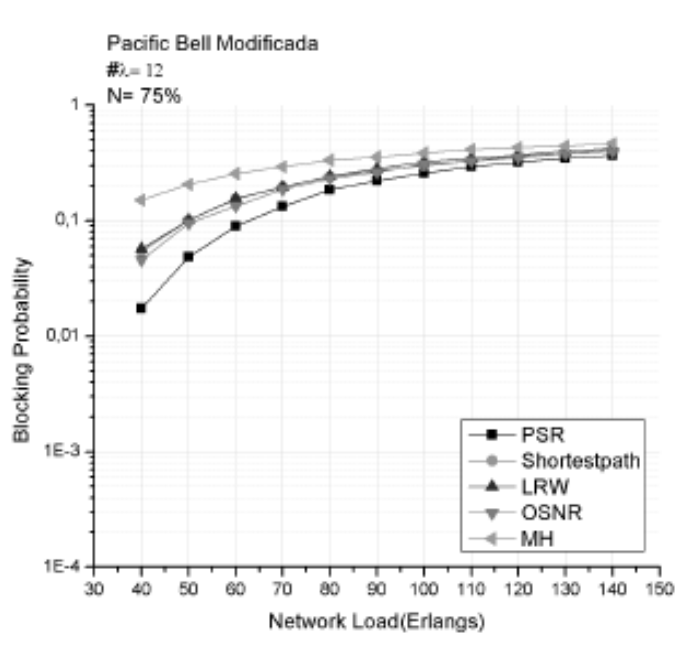

(a)

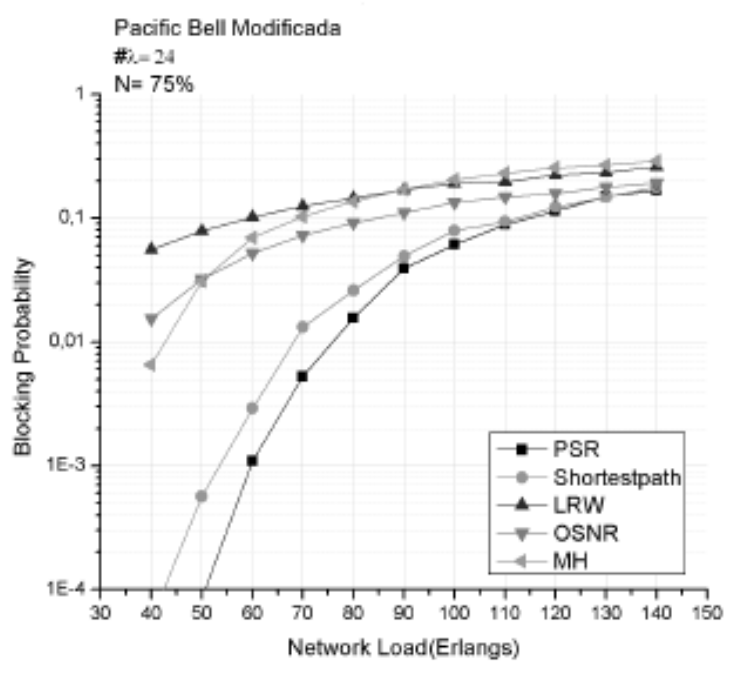

(b)

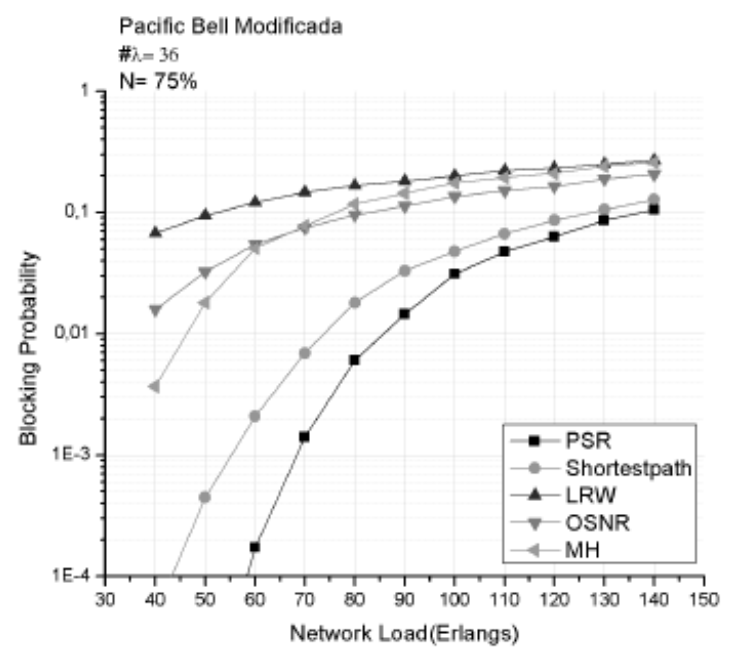

(c)

Figura 4: Topologia Pacific Bell Modificada considerando o cenário da rede translúcida com $75 \%$ dos nós translúcidos: (a) 12 comprimentos de onda, (b) 24 comprimentos de onda e (c) 36 comprimentos de onda.

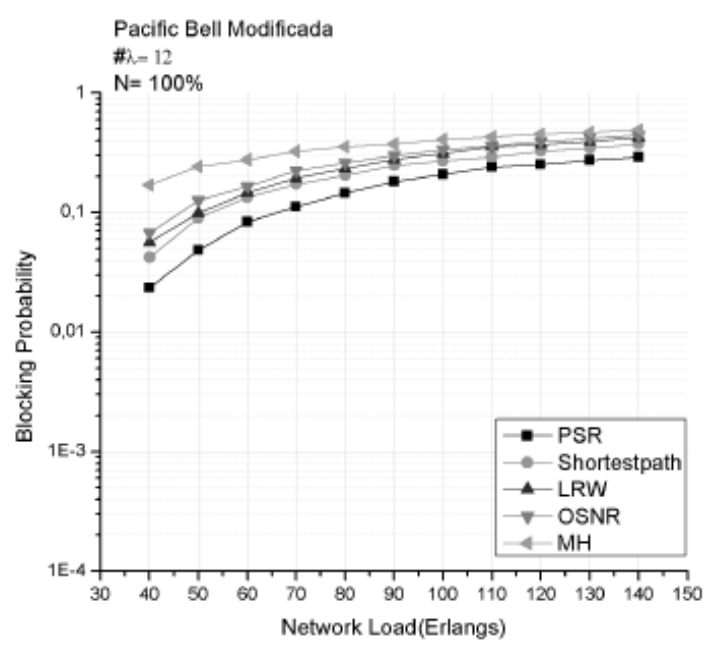

(a) 


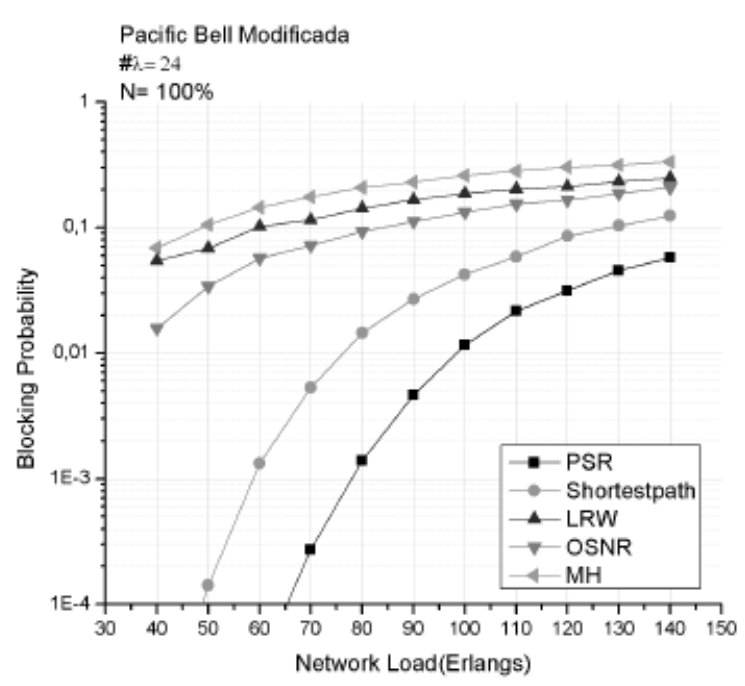

(b)

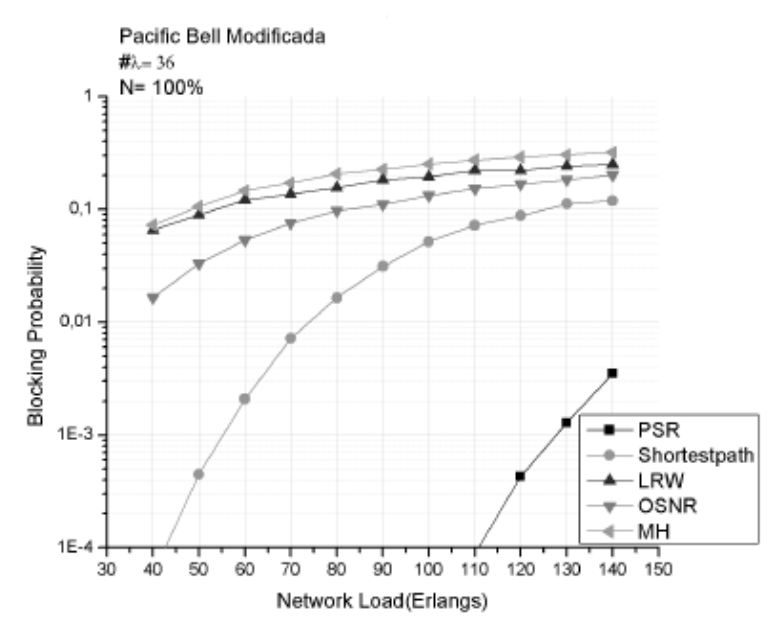

(c)

Figura 5: Topologia Pacific Bell Modificada considerando o cenário da rede opaca: (a) 12 comprimentos de onda, (b) 24 comprimentos de onda e (c) 36 comprimentos de onda.

\section{Conclusão}

Com as análises feitas dos algoritmos nesse trabalho, conclui-se que o algoritmo PSR, que havia sido implementado para redes ópticas transparentes, conseguiu se adaptar para redes translúcidas obtendo melhor desempenho em termos de probabilidade de bloqueio das chamadas em relação aos outros algoritmos analisados em todos os cenários de simulação investigados.

Pode ser observado ainda que o algoritmo OSNR apresenta desempenho similar ao PSR no cenário de rede óptica transparente, no entanto essa característica não foi observada para os demais cenários translúcidos considerados.

O algoritmo SP para alguns cenários, como para a rede translúcida com $75 \%$ de nós com capacidade de regeneração, obteve desempenho melhor que os outros algoritmos analisados se aproximando do desempenho do PSR, que foi algoritmo com melhor desempenho em todos os cenários investigados.

\section{Agradecimentos}

Agradeço ao programa do Pibic/Poli, FACEPE, UPE e UFPE pelos incentivos recebidos.

\section{Referências}

[1] Daniel A. R. Chaves, Caio F. C. L. C. Ayres, Renan V. B. Carvalho, Helder A. Pereira, Carmelo J. A. Bastos-Filho e Joaquim F. Martins-Filho. Submetido ao International Microwave and Optoelectronics Conference, IMOC, 2009.

[2] CHAVES, Daniel A. R.; BASTOS-FILHO, Carmelo J. A. ; MARTINS-FILHO, Joaquim F . Ferramenta Computacional para Simulação de Redes Ópticas Transparentes. Anais do MOMAG 2008. Florianópolis : SBMO, 2008. v. 1. p. 908-913.

[3] L. Wang et al., "A hybrid control architecture for connection management in translucent wdm networks," Anais do IEEE Global Telecommunications Conference - GLOBECOM, Dezembro 2008, pp. 1-6.

[4] D.A.R. Chaves; C.J.A. Bastos-Filho; J.F. Martins- Filho. Algoritmos Rápidos de IRWA para Redes Totalmente ópticas. PhD thesis, Engenharia Elétrica, Universidade Federal de Pernambuco,2008.

[5] H. A. Pereira, D. A. R. Chaves, C. J. A. BastosFilho, and J. F. Martins-Filho, "OSNR model to consider physical layer impairments in transparente optical networks," Photonic Network Communications, Setembro2008,online, ttp://dx.doi.org/10.1007/s11107-008-0178-2.

[6] B. Ramamurthy et al., "Translucent optical wdm networks for the next-generation backbone networks," Anais do IEEE Global Telecommunications Conference-GLOBECOM, vol. 1, 2001, pp. 60-64. 
[7] Ramaswami, R., Sivarajan, K.N.: Optical Networks: A Practical Perspective, $2^{\mathrm{a}}$ ed. MorganKaufmann, San Francisco (2002).

[8] G. Shen, W. V. Sorin, and R. S. Tucker, "Crosslayer design of ase-noiselimited island-based translucent optical networks," Journal of Lightwave Technology, vol. 27, no. 11, pp. 14341442, Junho 2009.

[9] J. F. MARTINS-FILHO, D. A. R. CHAVES, C. J. A. BASTOS FILHO, D. O. AGUIAR, Intelligent and Fast IRWA Algorithm based on Power Series and Particle Swarm Optimization. Anais do $10^{\circ}$ International Conference on Transparent Optical Networks, 2008, Atenas, Grécia. v. 1. p. 158-161. 